\title{
Uso de energía cinética para la obtención de energía eléctrica para señalamientos viales tipo leds y alumbrado publico aplicado en la Ciudad de la Venta, Tabasco
}

\section{Use of kinetic energy for the obtaining of electrical power for road signs type leds and public lighting applied in the City of la Venta, Tabasco}

\author{
MARTINEZ-VALERA, Elizabeth†* \& MUÑOZ-ROQUE, Micaela \\ Instituto Tecnológico Superior de Villa la Venta Tabasco \\ ID $1^{\mathrm{er}}$ Autor: Elizabeth, Martinez-Valera / ORC ID: 0000-0002-4036-7873 \\ ID $1^{\text {er }}$ Coautor: Micaela, Muñoz-Roque / ORC ID: 0000-0002-7946-0354
}

DOI: $10.35429 / J E E .2019 .9 .3 .10 .20$

Recibido 20 Marzo, 2019; Aceptado Junio 30, 2019

\section{Resumen}

Esta investigación aborda la generación de energía alternativa, para la implementación de semáforos, todos necesitamos el uso y aprovechamiento de la energía eléctrica como un instrumento de transformación en la comunidad de La Venta, Tabasco. Existe una nula conciencia del uso de los señalamientos viales, por parte de los auto conductores de esta Ciudad; es por eso que se propone diseñar e implementar una infraestructura técnica que permita utilizar y transformar la energía cinética del paso vehicular en zonas de tránsito. El principal objetivo es obtener la mayor cantidad de energía a partir del empleo de un multiplicador de velocidad, la inercia de un disco y un generador eléctrico. La energía potencial de un vehículo debido a su peso puede ser aprovechada a través de un mecanismo que la transforme en energía cinética y posteriormente a energía eléctrica y canalizarla para el aprovechamiento de suministro para señalamientos viales tipo LEDS y para alumbrado publico, Ayudando al entorno social y a nuestro planeta para no contaminarlo más.

Energía Cinética, Leds, Transformación

\begin{abstract}
This research deals with the generation of alternative energy, for the implementation of traffic lights, we all need the use and use of electric power as an instrument of transformation in the community of $\mathrm{La}$ Venta Tabasco. There is no awareness of the use of road signs, by the drivers of this City; that is why it is proposed to design and implement a technical infrastructure that allows to use and transform the kinetic energy of vehicular traffic in transit areas. The main objective is to obtain the greatest amount of energy from the use of a speed multiplier, the inertia of a disk and an electric generator. The potential energy of a vehicle due to its weight can be exploited through a mechanism that transforms it into kinetic energy and then into electrical energy and channel it for the use of supply for road signs type LEDS and for public lighting, Helping the social environment and to our planet so as not to contaminate it anymore.
\end{abstract}

Kinetic Energy, Leds, Transformation

Citación: MARTINEZ-VALERA, Elizabeth \& MUÑOZ-ROQUE, Micaela. Uso de energía cinética para la obtención de energía eléctrica para señalamientos viales tipo leds y alumbrado publico aplicado en la Ciudad de la Venta, Tabasco. Revista de Ingeniería Eléctrica. 2019. 3-9: 10-20

\footnotetext{
$\dagger$ Investigador contribuyendo como primer Autor.

*Correspondencia al Autor correo electrónico:(mave_sbc@hotmail.com)
} 


\section{Introduccion}

Desde el descubrimiento de la energía eléctrica el hombre ha buscado varias formas de producirla, ya que en esta nueva era de avances tecnológicos y científicos esta energía es esencial para el funcionamiento de muchos de los aparatos que utilizamos diariamente. La producción de esta energía ha ido avanzando con los años, pero aun dependemos mucho de los combustibles fósiles para obtenerla y ello conlleva a la contaminación del planeta, aunque en los últimos años se han desarrollado tecnologías no contaminantes para la producción de energía eléctrica, como ejemplos tenemos la Eólica, Solar, Mareomotriz, Hidráulica, etc.

El trabajo de investigación tiene como objetivo desarrollar el prototipo para la obtención de energía a través del tránsito vehicular por un reductor de velocidad, ubicado en las avenidas como una medida de seguridad vial, las autoridades vienen instalando reductores de velocidad, conocidos como "tope" con la intención de reducir la potencialidad de accidentes e incidentes de tránsito en los sectores de las carreteras que atraviesan las zonas urbanas y que no presentan la debida señalización.

En el país existen muchas estaciones de pasaje y de peaje que no cuentan con suministro de energía eléctrica por lo que recurren al uso de sus propios grupos electrógenos. En algunos casos, estas estaciones tienen un bajo consumo de energía, sin embargo, deben mantener encendido el grupo electrógeno, lo que significa un permanente consumo de combustible.

En muchas zonas urbanas el suministro eléctrico se realiza por cableado aéreo, lo que en algunas ocasiones resulta difícil e inseguro conseguir la electricidad para la señalización e iluminación diferenciada del reductor de velocidad.

El principal objetivo es obtener la mayor cantidad de energía a partir del empleo de un multiplicador de velocidad, la inercia de un disco y un generador eléctrico. La energía potencial de un vehículo debido a su peso puede ser aprovechada a través de un mecanismo que la transforme en energía cinética y posteriormente a energía eléctrica.
Este sistema neumático, eléctrico y electrónico ofrece una fuente de energía que podrá ser usada en señalamientos viales tipo leds (semáforos) en las carreteras, estaciones de pasaje y de peaje que no cuentan con el mismo; así como los "tope" ubicados en las zonas urbanas en el que el suministro de energía por la vía aérea resulta peligroso.

Este trabajo se genera con el objetivo de transferir tecnología que ayude el entorno social y a nuestro planeta para no contaminarlo, el diseño y mantenimiento de este sistema es sencillo, y nos confirma que la idea es factible y práctica. Teniendo proyección hacia el futuro, ya que se suma a las demás energías alternativas, renovables y limpias ya que presentan grandes ventajas en cuanto a su relación con el medio ambiente, su carácter inagotable y se determinan aspectos en los que se debe poner énfasis, primero la seguridad de las personas, así como la de los automovilistas.

\section{Base Teórica}

El suministro de energía es esencial para el funcionamiento de nuestra sociedad, tanto para la provisión y prestación de bienes y servicios, como en su faceta de factor productivo, que puede llegar a representar una de las claves de la competitividad de muchos sectores económicos. No hay duda de que la energía debe erigirse en un elemento dinamizador dentro de la economía y nunca llegar a convertirse en obstáculo para su crecimiento. (Paul ,2013).

En México la Comisión Federal de Electricidad (CFE), abastece de energía eléctrica al $97 \%$ de la población mexicana. La CFE genera, transmite, distribuye y comercializa energía eléctrica con alrededor de 900 mil nuevos clientes cada año, y a causa de la desaparición de Luz y Fuerza del Centro (LFC) que lo realizaba en el centro del país (D.F.) y área metropolitana.

Ahora la CFE abastece de energía eléctrica a la totalidad de país. La CFE ha unificado los criterios técnicos y económicos del Sistema Eléctrico Nacional, normalizando los voltajes de operación para estandarizar los equipos, reducir sus costos y los tiempos de fabricación, almacenaje e inventariado. 
Ha unificado también la frecuencia a 60 hertz en todo el país y se han integrado los sistemas de transmisión en el Sistema Interconectado Nacional. Aunque se supone que a través del Centro Nacional de Control de Energía (CENACE) se garantiza la seguridad, calidad y economía del suministro de energía eléctrica en el Sistema Eléctrico Nacional. (Satow 2009).

El uso de la energía mecánica para facilitar el desarrollo de actividades ha sido desde hace miles de años una forma de lograr más confort para el ser humano. La energía cinética del aire en movimiento se transforma en energía eólica y es entonces cuando puede ser aprovechada para mover elementos o dispositivos que transformen esa energía eólica en otro tipo de energías, de tal manera que pueda tenerse en la salida de un sistema otra forma de energía, normalmente la energía eléctrica.

El uso de la energía eléctrica ha traído grandes beneficios a la humanidad, pero también ha logrado que se contamine el medio ambiente por la utilización de combustibles fósiles que emiten tasas muy altas de agentes contaminantes a la atmósfera. Esto ha generado una preocupación mundial por buscar nuevas fuentes de energía que no contaminen y cuyo costo de generación sea competitivo. (Ruedas 2011).

La generación de energía eléctrica es tal vez una de las principales fuentes de desarrollo y de mejoramiento de la calidad de vida del hombre actual, ya que, gracias a ella, hoy en día es posible llevar a cabo un sin número de actividades que contribuyen al crecimiento integral de la sociedad, tanto desde el punto de vista científico y tecnológico, como industrial, cultural y económico. Por esta razón, la energía eléctrica se ha convertido en uno de los servicios sociales de mayor demanda e importancia en nuestro medio, haciéndose cada vez más indispensable para la ejecución de actividades de gran trascendencia.

En general puede decirse que la energía de origen hidráulico ha sido la de mayor acogida hasta el momento, a pesar del surgimiento de otras formas de obtención de energía eléctrica a partir de diversas fuentes de generación, tales como la energía nuclear, la eólica, la solar, entre otras.
Y debido a esa gran aceptación o respuesta que se logró de la generación hidroeléctrica (por facilidades de construcción, economía, impacto ambiental y costos de mantenimiento, pues utiliza como materia prima un recurso renovable), y a la importancia de la electricidad, cada vez se ha vuelto más especializado el estudio de este proceso, convirtiéndose en un amplio campo de acción de la ingeniería.

En cada instante en el universo se convierte energía mecánica en eléctrica. Sin pretensión de entrar en consideraciones sobre la diferencia entre un tipo de energía y otra, que, sin duda, cuanto más se profundice sobre la estructura del universo y de lo que lo compone menor es, se producen a todos los niveles procesos en los que la energía cinética de una masa pasa a ser, con una cadena de conversiones más o menos compleja, energía eléctrica. (Jurado 2007)

La energía no es un concepto fácil de asimilar ya que realmente la energía sólo se percibe en la medida en que realiza trabajo. Podríamos definir energía como la capacidad de producir trabajo. Pero, ¿qué pasa con la energía una vez que se ha producido el trabajo? De acuerdo con el Primer Principio de la Termodinámica, la energía ni se crea ni se destruye, sólo se transforma. Por tanto, cuando se produce un trabajo, la energía se degrada, convirtiéndose en algo incapaz de realizar trabajo. La energía se puede presentar de diversas formas: energía mecánica, térmica, química, nuclear, electromagnética.

Cuando se habla de fuentes de energía, se distingue entre energía primaria, energía secundaria y energía final. La diferencia radica en que la energía final es aquella que se utiliza directamente como trabajo o calor, mientras que la energía primaria es la obtenida directamente de la naturaleza, contenida en las materias primas energéticas y que necesita ser transformada en energía secundaria o intermedia antes de su consumo final. La energía secundaria es energía mecánica, eléctrica o térmica.

\section{La creciente demanda de energía}

Según el Centro Público Pablo Guzmán, la nueva sociedad que nació de la Revolución Industrial trajo también nuevas demandas de energía.

MARTINEZ-VALERA, Elizabeth \& MUÑOZ-ROQUE, Micaela. Uso de energía cinética para la obtención de energía eléctrica para señalamientos viales tipo leds y alumbrado publico aplicado en la Ciudad de la Venta, Tabasco. Revista de Ingeniería Eléctrica. 2019 
Con la máquina de vapor aparecieron inventos revolucionarios que mejoraron los medios de transporte, como la locomotora que George Stephenson construyó en 1825.

Sin embargo, a pesar de que este sistema de locomoción era seguro y eficaz, consumía grandes cantidades de carbón para convertir la energía calorífica en mecánica; el rendimiento que producía era inferior a un $1 \%$. Aún hoy día se consume gran cantidad de energía para producir un rendimiento muy inferior; por ejemplo, una central eléctrica que utilice carbón o petróleo rinde menos del $40 \%$, y en el caso de un motor de combustión interna incluso menos del $20 \%$.

Esta pérdida de rendimiento es a causa de las leyes físicas; la energía que no utilizamos (o no somos capaces de aprovechar) no se pierde, sino que se transforma; en los casos de combustión interna, por ejemplo, el resto de energía que no aprovechamos se disipa en forma de calor. Por ello, una lucha tecnológica constante es la de mejorar el rendimiento de las máquinas para aprovechar al máximo la energía.

La neumática se encarga del estudio de las propiedades y aplicaciones de los gases comprimidos, Aunque las aplicaciones de los fluidos (gases) no son nuevas, lo que sí es relativamente reciente es su empleo en circuitos cerrados en forma de sistemas de control y actuación. Un problema de automatización y control puede resolverse empleando mecanismos, circuitos eléctricos y electrónicos, circuitos neumohidráulicos $\mathrm{o}$ bien una combinación de todo ello.

\section{Circuito neumático}

Los circuitos neumáticos utilizan aire sometido a presión como medio para la transmisión de una fuerza. El aire se toma directamente de la atmósfera y se deja salir libremente al final del circuito habitualmente través de un silenciador, pues de lo contrario resultan muy ruidosos. $\mathrm{La}$ distancia desde el depósito hasta el final del circuito puede ser de decenas de metros.

\section{Ventajas del aire comprimido:}

Es abundante (disponible de manera ilimitada).
Transportable (fácilmente transportable, además los conductos de retorno son innecesarios).

- Se puede almacenar (permite el almacenamiento en depósitos).

- Resistente a las variaciones de temperatura.

- Es seguro, antideflagrante (no existe peligro de explosión ni incendio).

Limpio (lo que es importante para industrias como las químicas, alimentarias, textiles, etc.).

- Los elementos que constituyen un sistema neumático, son simples y de fácil comprensión).

- $\quad$ La velocidad de trabajo es alta.

- Tanto la velocidad como las fuerzas son regulables de una manera continua.

\section{Ventajas de la neumática}

- La neumática resulta útil para esfuerzos que requieran precisión y velocidad.

- $\quad$ Aguanta bien las sobrecargas (no existen riesgos de sobrecarga, ya que cuando ésta existe, el elemento de trabajo simplemente para sin daño alguno).

Emprendedores mexicanos han desarrollado un sistema que aprovechará el tráfico de vehículos para generar energía eléctrica. Ese desarrollo tiene la potencialidad de producir la electricidad necesaria para alimentar un hogar a través de un dispositivo que "atrapa" la fuerza de los automóviles en movimiento.

"Se trata de una tecnología que provee energía sostenible y podría resultar bastante económica, pues es un complemento a la infraestructura ya existente: el hormigón de las calles y avenidas", destaca Héctor Ricardo Macías Hernández, desarrollador del sistema, que agrega que a nivel mundial no existen antecedentes de proyectos similares, con excepción de una patente inglesa, pero con la diferencia de que en ese país europeo se emplean pisos piezoeléctricos, que resultan demasiado costosos para países en desarrollo.

La tecnología consiste en un sistema que integra una rampa-escalón (elaborada a partir de material polimérico similar al de las llantas) que se eleva a cinco centímetros sobre el nivel del concreto. Al recibir el impacto provocado tanto por el tonelaje como por la inercia del vehículo, esa rampa ejerce presión sobre un fuelle.

MARTINEZ-VALERA, Elizabeth \& MUÑOZ-ROQUE, Micaela. Uso de energía cinética para la obtención de energía eléctrica para señalamientos viales tipo leds y alumbrado publico aplicado en la Ciudad de la Venta, Tabasco. Revista de Ingeniería Eléctrica. 2019 
Este artefacto, al contener aire, lo expele a cierta presión a través de una manguera; posteriormente, ese elemento viaja hacia un tanque que lo comprime y lo relanza a una turbina generadora de electricidad. Macías Hernández comenta que la acumulación de energía eléctrica es proporcional al flujo de automóviles que existe sobre determinado lugar; sin embargo, explica que en un lugar con poca afluencia vehicular podrían ser colocadas varias rampas-escalón para multiplicar el impacto del paso del vehículo.

La tecnología también podría ser implementada en lugares con alto flujo peatonal. De esa forma, los pasos de las personas estarían generando electricidad con base a las leyes de la energía gravitacional. Aprovechar el peso vehicular y peatonal para generar energía es una alternativa viable y sostenible. Israel muestra avances concretos en esta materia con la utilización de aplicaciones piezoeléctricas, mientras que en México se están delineando soluciones basadas en leyes de energía gravitacional.

Las soluciones piezoeléctricas no contaminan, la energía obtenida es renovable y sostenible, y su aplicación es adaptable a cualquier lugar, independientemente del clima y de la región geográfica. Utilizar la energía mecánica que generan los ferrocarriles durante su funcionamiento, o el paso de vehículos por carreteras y de paseantes por sendas peatonales, $\mathrm{y}$ aprovecharla para convertirla en electricidad es una opción que muchos países ya están adoptando.

En algunos lugares del mundo, se emplean soluciones basadas en el fenómeno físico de la piezoelectricidad, descrito por primera vez por los hermanos Pierre y Jacques Curie, a finales del siglo XIX. Otros investigadores se valen de las leyes de la energía gravitacional, como es el caso del investigador mexicano Héctor Ricardo Macías Hernández, para generar energía eléctrica.

\section{Carreteras, fuente potencial de energía}

En los automóviles, sólo una parte de la energía procedente de la combustión se utiliza para moverlo por la carretera o poner en funcionamiento algunos accesorios útiles, como el aire acondicionado.
El resto de la energía, debido a la ineficiencia de los motores, se pierde. Los generadores de Innowattech, que comenzaron a comercializarse en 2009 , se incrustan a una profundidad de alrededor de 5 centímetros y logran captar la energía de los vehículos cuando pasan por encima de ellos para reutilizarla. Este sistema, que es aplicable en asfalto, hormigón o cemento compuesto, no daña la carretera y no aumenta el consumo de combustible de los vehículos.

De acuerdo con estudios de Innowattech, el paso de 600 vehículos viajando a un promedio de 72 kilómetros por hora sobre una carretera con generadores piezoeléctricos instalados a lo largo de 1 kilómetro permitiría producir 200 kilowatts por hora.

\section{Desarrollo de la propuesta $y$ caso de aplicación}

De acuerdo a la naturaleza del estudio de la investigación, reúne por su nivel las características de un estudio descriptivo, explicativo y correlacionado.

El Estudio que se realiza sobre la generación de energía, mediante la hidráulica, se realiza mediante la consulta de material bibliográfico y hemerográfico, el cual nos llevó al diseño del prototipo para la realización del tope así también se realiza un sondeo de opiniones en el que se consulta directamente acerca de la generación de energías para la señalización por medio de semáforos leds.

La población motivo de esta investigación está conformada por 8,821 habitantes de la Venta Tabasco de todos los niveles los cuales se verán beneficiados por la implantación de señalamientos viales tipo leds y alumbramiento de calles.

La muestra a utilizar en esta investigación es todas aquellas personas que cuentan con un transporte ya sea de automóviles, motos, bicicletas, bicitaxis, etc. Así como cualquier otro tipo de persona que tenga interés por participar. 
Sistema Mecanico Hidraulico

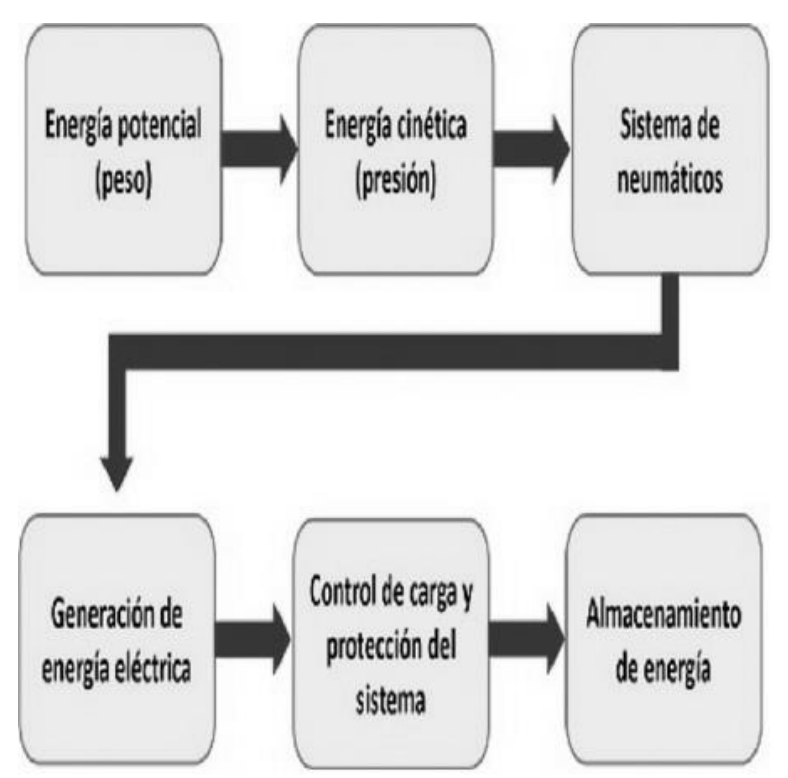

Figura 1 Diagrama de funcionamiento del sistema Mecanico-Hidraulico

Fuente: Elaboración Propia

En la figura 1, la energía potencial que entrega el peso del vehículo se aprovecha para la generación de energía eléctrica, la que es optimizada mediante el uso de un sistema de neumáticos. Se desarrolla un sistema mecánico hidráulico a ser instalado en un reductor de velocidad de tipo tope.

El paso de un vehículo por el "tope" desplazará la rampa del sistema hacia abajo transmitiendo el movimiento a unos actuadores neumáticos que accionará un sistema de generación de aire comprimido. El uso de un sistema de actuadores neumáticos tiene como finalidad generar aire para mover una turbina la cual genera energía multiplicar la velocidad para alcanzar la deseada en el generador eléctrico seleccionado.

Resortes para el retorno de la rampa: Estos resortes son fabricados de acero ASTM A229 y cuentan con un diámetro de alambre de $7 \mathrm{~mm}$, diámetro exterior de $35 \mathrm{~mm}, 2$ espiras activas y una constante de $130689.38 \mathrm{~N} \mathrm{~mm} / \mathrm{rad}$. Para esta selección se ha tenido en cuenta las cargas que estarán presentes, así como la constante equivalente que permite el retorno de la rampa a su posición inicial. Son 5 juegos de resortes que se encuentran distribuidos linealmente en las bisagras de la rampa del sistema. Es importante resaltar que los resortes no van a soportar el peso del vehículo, solo almacenaran la energía necesaria para poder retornar la rampa a su posición inicial.
Acumuladores de energía eléctrica: es un dispositivo que almacena energía por procedimientos electroquímicos y de la cual la energía se puede disponer en forma de electricidad. Los acumuladores se basan en la fuerza electromotriz (f.e.m.) de polarización que se crea durante un proceso de electrolisis y que se opone a la f.e.m aplicada para llevar a cabo esa electrolisis.

Batería principal: será usada para almacenar la energía generada. Esta batería es capaz de suministrar $480 \mathrm{Wh}$, sin embargo, para este diseño sólo se ha considerado emplear el 85 $\%$ de su capacidad de carga (408 Wh). La tecnología de esta batería es del tipo Li-Ion, se escogió esta batería debido a que cuenta con características del tipo LiFePo4 (indicado por el fabricante) porque es capaz de recibir grandes cantidades de corriente de carga, por lo general entre $0,5 \mathrm{C}$ y $3 \mathrm{C}$. La batería seleccionada soporta como máxima corriente de carga $1 \mathrm{C}$, es decir 40 A. Además, la batería cuenta con un sistema de protección interno contra cortocircuitos, sobrecargas y sobre descargas.

La batería secundaria seleccionada es del tipo VRLA (batería de ácido-plomo regulada por válvula) y sólo será utilizada como fuente de alimentación para el circuito de control de carga. Se ha considerado el uso de esta batería para mantener una fuente constante en el circuito de control, debido a que se espera que la batería principal se descargue continuamente. No se requiere que esta batería soporte una gran cantidad de corriente de carga, debido a que su corriente de descarga es pequeña $(1.97 \mathrm{~mA})$. Se mantendrá cargada con una corriente mayor a la corriente de flotación indicada por el fabricante, debido a que la carga no es constante. Para evitar la sobrecarga de la batería, se ha previsto el uso de un diodo Zener y un transistor en paralelo.

\section{Ecuaciones y Cálculo de generación de energía eléctrica}

El proyecto utilizara alternadores de $12 \mathrm{~V}$ de C.A. Y $5.5 \mathrm{~W}$ de generación. Se debe de calcular la corriente que genera para poder hacer una comparación con las especificaciones de la luminaria a utilizar

$$
\mathrm{I}=\frac{5.5 \mathrm{~W}=0.4435 \mathrm{~A}}{12 \mathrm{~V}}
$$


Teniendo una corriente producida de 0.4435 Amperes. Si la velocidad del rotor por cada golpe de automóvil sea de 2500 RPM.

NOTA: La velocidad se obtuvo a partir de experimentos realizados para obtener la tensión de $12 \mathrm{~V}$

$\mathrm{KW}=\left(\begin{array}{c}600 \\ \text { automóviles }\end{array}\right)\left(\begin{array}{c}5.5 \mathrm{~W} \text { por } \\ \text { dinamo }\end{array}\right)=3.3 \mathrm{Kwh}$

Una vez teniendo esto consideramos que la generación con un alternador de CA. Se debe alimentar una carga aproximada de $120 \mathrm{kWh}$ ¿Por qué? En aproximadamente una concurrencia de 600 automóviles distribuidos uniformemente.

\section{Entonces se tiene que:}

Para poder calcular el porciento de error en la generación que hay que alcanzar se debe considerar que se debe sobre dimensionar un poco la capacidad generada del proyecto si consideramos un porciento de error de $10 \%$ para una generación de $120 \mathrm{kWh}$ obtenemos que lo ideal es que se produzcan $132 \mathrm{~kW}$ de energía o más.

$$
\begin{aligned}
& 132 \mathrm{~kW}-120 \mathrm{~kW}=12 \mathrm{KW} \text { sobredimensionado } \\
& \mathrm{kWs}=\frac{12 \mathrm{~kW}}{2}=6 \mathrm{~kW}
\end{aligned}
$$

El proyecto deberá de estar sobredimensionado +- $6 \mathrm{~kW}$

$$
\begin{aligned}
& \begin{array}{l}
\% \text { error en } \\
\text { generación }
\end{array}=\frac{126 \mathrm{kw}-120 \mathrm{kw}}{126 \mathrm{kw}} \begin{array}{l}
=5 \% \text { de } \\
\text { error abajo }
\end{array} \\
& \begin{array}{l}
\% \text { error en } \\
\text { generación }
\end{array}=\frac{132 \mathrm{kw}-126 \mathrm{kw}}{132 \mathrm{kw}}=\begin{array}{c}
5 \% \text { de error } \\
\text { arriba }
\end{array}
\end{aligned}
$$

De acuerdo con lo calculado la capacidad de generación se puede alcanzar y superar con aproximadamente 600 vehículos. Estos para que la generación sea eficiente deberán tener sobre dimensionado $5 \%$ de la generación mínima $120 \mathrm{kWh}$ dando la capacidad de 126kWh. Esto debido a que la concurrencia de los automóviles no siempre será la esperada.
Los cálculos realizados anteriormente tienen la intensión de analizar la capacidad de generación que tiene un solo generador eléctrico y comparándolo con el proyecto que ya existe de características similares.

Esa capacidad de generación debe de ser adecuada para poder alimentar un número de luminarias con las características que antes se especificaron de las cuales las principales son: $60 \mathrm{Wh}$ de consumo $24 \mathrm{~V}$.

También se considerará que una luminaria de leds se debe de alimentar a una tensión de $24 \mathrm{v}$ y una potencia de $60 \mathrm{Wh}$ entonces la corriente que necesita la luminaria será de.

$\mathrm{I}=\frac{(60 \mathrm{~W} \mathrm{~h})(.18)}{24 \mathrm{v}}=.444 \mathrm{~A}$

El $18 \%$ es el factor de corrección que utilizado para poder calcular la corriente que consume una luminaria led este factor de corrección es aplicado ya que la corriente que consume el led es menor al de cualquier otro tipo de luminaria

Como se observa en la ecuación anterior la corriente que se debe de producir al momento de que gire el alternador debe de ser de 0.444A, sin embargo, la tensión no es la adecuada para poder alimentar el luminario es por esto que se debe de llegar a los valores nominales por medio de arreglos de generadores.

El resultado anterior fue la capacidad producida por un solo par de generadores que solamente representa una pequeña parte de la capacidad que se requiere para igualar el proyecto ya existente considerando que deben de pasar al menos $600 \mathrm{~W}$ en una hora.

Se debe saber cuánto dinamos de CA se necesitan para producir la capacidad de $120 \mathrm{kWh}$ a superar o por lo menos igualar.

De cálculos anteriores se sabe que 600 automóviles crean $132 \mathrm{~kW}$ con un solo par de alternadores además de que esta potencia es generada por el paso de los 2 ejes de los automóviles que son el delantero y el trasero. 
Como un solo par de generadores no es suficiente entonces se debe de tener un mayor número de estos para que se aporte una mayor cantidad de kWh al sistema. Esto tiene solución si se tienen 10 pares de generadores aportando energía al mismo tiempo a un grupo de baterías que puedan almacenar toda la energía para que las luminarias la consuman.

$$
k W h \text { totales }=132 k W h * 10=1320 K W h
$$

\section{Diseño de la rampa o del sistema Mecanico- Hidraulico}

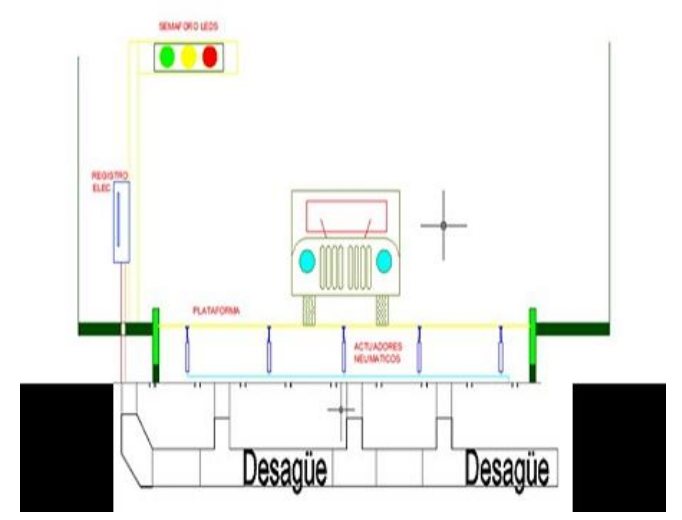

Figura 2 Diseño del sistema Fuente: Elaboración Propia

\section{Conformación del Sistema}

$\begin{array}{ll}- & \text { Rampa o tope } \\ - & \text { Actuadores } \\ - & \text { Tanque } \\ - & \text { Turbina } \\ - & \text { Banco de baterías } \\ - & \text { Registro de flujo } \\ - & \text { Resorte de Alta Resistencia }\end{array}$

Vista lateral del ensamble general del sistema mecanico-hidraulico.

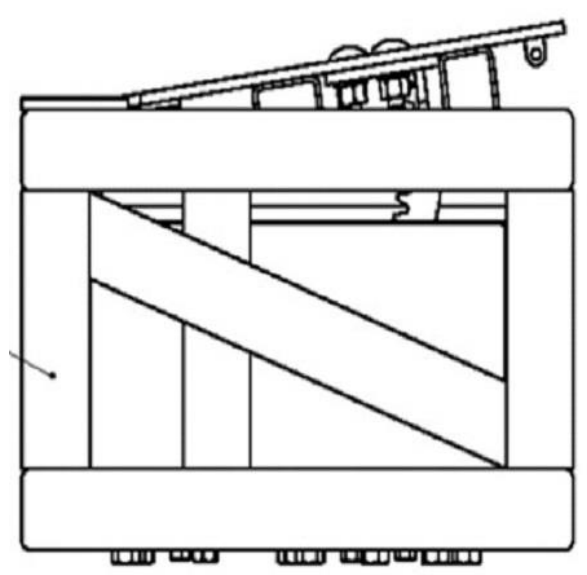

Figura 3 Vista Lateral

Fuente: Elaboración Propia

\section{Sistema de desagüe}

El sistema desagüe que tendrá el proyecto es parte fundamental del anteproyecto ya que no todas las calles son uniformes y aunque así sea debido a que hay partes que suben y bajan del nivel del piso hay filtración de agua y polvo.

Estos factores que no se pueden controlar pueden afectar, de tal modo que de acuerdo al lugar se tendrá que estudiar estadísticas del comportamiento ambiental, y de esta forma proponer un plan de mantenimiento.

El sistema de desagüe estaría conectado a el sistema de drenaje de la ciudad en caso de tenerlo y su implementación se muestra en la figura, en caso de no contar con él, lo que se necesitaría hacer es sacar el tubo de drenaje de agua hacia las áreas verdes más cercanas y que la salida no se obstruya con nada entonces se tendría que construir una pequeña coladera o registro.

\section{Material a Utilizar para sistema mecanico- hidraulico}

El material que se utiliza para la estructura del tope es de uso rudo es acero al alto carbono esto por su dureza y densidad, para el proyecto estas características son esenciales puesto que tendrá un esfuerzo mecánico muy grande provocado por el golpe del paso de los automóviles.

Acero para herramientas: ACERO 4840R este material es de fácil temple y es usado para fabricar partes mecánicas sujetas a un gran esfuerzo mecánico como pueden ser engranes, engranes sin fines, flechas, piñones, etc. Este tipo de material nos ayudara a evitar el desgaste mecanico rápidamente. La composición del acero en general para este tipo de herramientas debe ser: carbono $(.38-.43 \%)$, silicio $(.15$ $.35 \%)$, manganeso $(.79-.90 \%)$, potasio $(.04 \%)$, sulfuro $(.04 \%)$, cromo (.70 - .90\%), níquel (.85$1.15 \%)$.

\section{Plan de mantenimiento}

En el plan de mantenimiento se planteará conforme a cómo se comporta el clima en la República Mexicana Específicamente en el Estado de Tabasco ya que es ahí donde se implantará específicamente en la ciudad de la Venta, Tabasco. 
Es importante saber esta información ya que es preferible checar las condiciones del mecanismo en fechas más peligrosas para el mismo que es al estar a mitad de periodo de lluvias e inmediatamente después de terminar temporada de lluvias. El siguiente plan de mantenimiento es preventivo y las partidas a revisar del mecanismo son las siguientes:

1. Revisión de funcionamiento del desagüe (desazolvar en caso de ser necesario).

2. Que tenga una falta de lubricación (lubricar en caso de ser necesario).

3. Ver que el metal contenga un bajo nivel de oxidación. (limpiar en caso de ser necesario).

4. Revisar la condición de desgaste de las piezas (en caso de necesitar cambio de piezas el tiempo varía de acuerdo a las mismas).

5. Pruebas eléctricas a generadores (Cambio de los mismos, el tiempo está sujeto a cambios de acuerdo al número de elementos a cambiar).

\section{Factibilidad:}

Este proyecto se genera con el objetivo de transferir tecnología que ayude el entorno social y a nuestro planeta para no contaminarlo, el diseño y mantenimiento de este sistema es sencillo, y nos confirma que la idea es factible y práctica.

Teniendo proyección hacia el futuro, ya que se suma a las demás energías alternativas, renovables y limpias ya que presentan grandes ventajas en cuanto a su relación con el medio ambiente, y su carácter inagotable, se determinan aspectos en los que se debe poner énfasis, primero la seguridad de las personas, así como la de los automovilistas.

En la actualidad un semáforo tradicional gasta alrededor de $1200 \mathrm{~W}$ por hora lo cual genera un gasto $10,713,600.00 \mathrm{~W}$ por año, con un costo de $\$ 79,442.624$ anual mente por semáforo.

Comparando con el precio del tope lo cual es de $\$ 58,790.00$ lo cual solo necesitara un mantenimiento mínimo y cuenta con una duración de 15 años de vida útil tendremos un ahorro de $\$ 1,132,849.36$ aproximadamente lo cual lo hace un proyecto altamente rentable.

\section{Ubicación del mecanismo}

Como se mencionó la ubicación de este mecanismo es en la ciudad de la Venta Tab., debe de ser la correcta tiene que haber una concurrencia de 600 automóviles. Se tendrá actuadores neumáticos que generan según los cálculos efectuados individualmente para producir 47.99W aproximadamente con una tasa promedio de 600 Automóviles se producirán $287.982 \mathrm{~W}$ y se tiene pensado tener 6 actuadores neumáticos entonces la generación total en Watts sería de 172789 kWh esto con un eje de automóvil.

La importancia de estar en un lugar apropiado que necesite iluminación sin embargo que cuente con un poco de infraestructura que carezca de mantenimiento como postes reduciría el costo del proyecto y a su vez que en dado caso de interrupción de paso en la vía tenga rutas alternas o un tránsito por calles aledañas. En tanto se terminen las construcciones del proyecto.

\section{Resultados}

A la fecha se cuenta de manera física con un simulador a escala que nos proporcione una visión clara y precisa de que el sistema funciona, almacenando la energía en un banco de baterías para posteriormente ser utilizadas en la implantación de semáforos, con este simulador hemos logrado alcanzar 40lb de presión con el peso de una persona y se ha logrado prender los leds, por lo cual consideramos que el sistema es factible y rentable, su mantenimiento es sencillo ya que ninguno de los elementos que componen el sistema requieren mantenimiento especializado.

La Venta, es una ciudad del estado mexicano de Tabasco, siendo la segunda más importante del municipio de Huimanguillo, el incremento en el número de vehículos ha hecho que la urbe misma se modifique, porque donde hace décadas había calles o avenidas con grandes camellones llenos de árboles o palmeras, ahora hay ejes viales, autopistas y avenidas.

Esto nos lleva a que la demanda de automóviles es grande por año esto nos da un amplio panorama de que se puede generar gran cantidad de energía renovable y no contaminada por lo que no se cuenta con competidores de esta índole.

MARTINEZ-VALERA, Elizabeth \& MUÑOZ-ROQUE, Micaela. Uso de energía cinética para la obtención de energía eléctrica para señalamientos viales tipo leds y alumbrado publico aplicado en la Ciudad de la Venta, Tabasco. Revista de Ingeniería Eléctrica. 2019 
Cabe destacar que este prototipo a participado en varias convocatorias obteniendo el ler lugar como es:

\section{- 3er concurso estatal innovando energía en el estado de Tabasco \\ $2^{\circ}$ Foro de Divulgación Científica y Tecnológica nov. 2014 en el ITSLV.}

También ha participado en el Congreso Internacional de la Academia Journals Celaya 2014.

\section{Conclusión}

Para el desarrollo del sistema generador de corriente eléctrica y de manera sustentable, se tiene la necesidad de buscar una energía alternativa no contaminante y aprovechar la energía cinética que genera el tráfico vehicular que transita, para utilizarla en señalamientos viales, ya que tiene como objetivo tener un beneficio social, cultural y tecnológico.

El instituto Tecnológico será el primero en instalar este tipo de modernos Semáforos Inteligentes además de ser el primero de su tipo instalados en Tabasco, esta nueva tecnología en controladores de tránsito que poseen mayor durabilidad que alcanza 100 mil horas frente a 8 mil de un bombillo convencional y nos permite tener 100 veces menos consumo de energía para su funcionamiento.

La tecnología LED se fundamenta en la programación computarizada de bombillos individuales con mayor grado de luminancia que un bombillo convencional. Por su diseño, en caso de dejar de funcionar un bombillo no se apaga la cara completa del semáforo, sino uno de los tantos bombillos identificadores y por su versatilidad permite dibujar diferentes figuras sin necesidad de usar otras piezas para definirlas, lo cual genera ahorros en el mantenimiento preventivo anual.

De lo anteriormente expuesto se toma como base de innovación la instalación de diodos tipo LED que reducen 100 veces el consumo normal de un semáforo con iluminación por lámparas, disminuyendo la demanda de energía para su funcionamiento lo cual hace de este sistema de gran utilidad.
Este sistema recolector de energía sin moverse de lugar será capaz de generar tanta energía que será necesaria almacenarla en bancos de baterías para su posterior utilización, ya que el tránsito vehicular permanece constante las veinticuatro horas y además se intensifica en ciertas horas del día.

El esquema mecánico cumple con los requerimientos tanto de fácil montaje al suelo para una construcción $\mathrm{y}$ acceso rápido al momento de dar mantenimiento. A demás de contar con un sistema de desagüe para evitar encharcamientos en épocas de lluvia y finalmente tiene un plan de mantenimiento preventivo para asegurar su durabilidad, así el proyecto cumple con requisitos esperados.

\section{Referencias}

Camilo González Paul. (2013). Energías renovables no convencionales para uso domiciliario. Santiago, chile: universidad de chile facultad De Derecho Departamento De Derecho Económico.

Crane, 1987, "Flujo de fluidos en válvulas, accesorios y tuberías", McGraw-Hill.

Edgar López Satow. (2009). Utilización De Energías Renovables En México: Hacia Una Transición En La Generación De Energía Eléctrica México: Universidad Nacional Autónoma De México.

Francisco Bañuelos Ruedas. (2011). Impacto De La Generación Eléctrica Usando Fuentes De Energía Eólica En La Red Eléctrica Nacional. México: Universidad Nacional Autónoma De México.

González, J., Ballesteros, R., Parrondo, J. L., 2005, "Problemas de oleohidráulica y neumática", Servicio de Publicaciones de la Universidad de Oviedo.

José Antonio Aguilera Folgueiras. (2012). Fuentes de energía y Protocolo de Kioto en la Evolución del Sistema Eléctrico Español. Oviedo: UNIVERSIDAD DE OVIEDO.

Julio Andrés Cáceres Vergara. (2006). Estudio De Pre-Factibilidad De La Microcentral Hidroeléctrica De San Pedro De Huacos - Canta - Lima. Lima - Perú: Universidad Nacional De Ingeniería.

MARTINEZ-VALERA, Elizabeth \& MUÑOZ-ROQUE, Micaela. Uso de energía cinética para la obtención de energía eléctrica para señalamientos viales tipo leds y alumbrado publico aplicado en la Ciudad de la Venta, Tabasco. Revista de Ingeniería Eléctrica. 2019 
Lavonbille, R., 1991, "Circuits hydrauliques". Ecole Politechnique de Montreal (Canadá). SMC, 2000, "Neumática", Thomson-Paraninfo.

Manuel Pinilla Martín (2011). Sistema de conversión electromecánica de alta potencia específica para generación eléctrica de origen renovable. Madrid: universidad politécnica de Madrid.

Marcelo Betancourt jurado. (2007). Diseño de un módulo interactivo de generación hidráulica de energía eléctrica. Pereira: Universidad Tecnológica De Pereira.

White, F.M., 2004 (quinta edición), "Mecánica de Fluidos", McGraw-Hill. 